\title{
Interview avec Peter Müller-Locher, membre de l'ASP
}

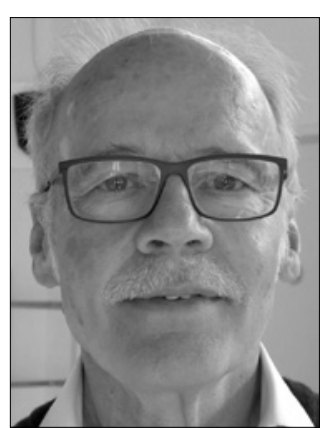

Peter Müller-Locher
Qu'est ce qui vous a motivé à choisir la profession de psychothérapeute?

Mon père est allé voir un psychothérapeute théologique parce que, à l'âge de 18 ans, j'avais quitté le club de handball confessionnel en faveur d'un club plus attrayant. Le théologien jungien m'a convoqué, a fait preuve de compréhension, a soutenu mon choix et m'a relativement expliqué l'inquiétude de mon père. Un an plus tard, jai décidé d'étudier la psychologie après l’obtention de mon diplôme. Je voulais aussi devenir psychothérapeute, mais pas principalement jungien. Je décris également ce passage dans un travail biographique enrichi (2011). Cela peut montrer, à titre d'exemple, qu'une expérience personnelle utile pour choisir une carrière de psychothérapeute peut être une motivation cruciale.

Quel est votre parcours/expérience professionnel? J’ai étudié la psychologie, la psychopathologie et la philosophie à l'université de Zurich. Après deux ans comme professeur d'école secondaire sans formation, j'ai ouvert mon cabinet en tant que conseiller psychologique et jai commencé la première formation de psychothérapie en Daseinsanalyse.

Travaillez-vous en tant que psychothérapeute indépendant en cabinet privé et/ou travaillez-vous (éventuellement en plus) en tant que psychothérapeute délégué ?

Dans les années 1970, d'une part, dans le canton de Zurich, loffre de psychothérapie par des psychologues était toujours illégale au sens de la loi sur la santé, mais cétait une pratique courante. D’autre part, faire de la psychothérapie était encore plus souvent considéré comme une affaire d'émancipation et pas seulement comme un soi-disant traitement médical. Et troisièmement, le concept de psychothérapie déléguée n'existait pas encore. Dans le cadre de ces conditions, j’ai réussi à exercer et à préserver ma profession en tant que profession indépendante - aujourd'hui avec la licence nécessaire pour exercer, en outre avec un accent mis sur le développement de la personnalité et sans implication dans l'assurance de base

Y a-t-il une autre profession, une autre occupation, que vous faites en plus de la psychothérapie?

Un tiers de mes offres de service appartiennent au domaine de la supervision (analytique et systémique) dans divers contextes. Ma future quali- fication en tant que développeur organisationnel a été fructueuse au sein de la Commission pour l'assurance qualité de la Charte Suisse de psychothérapie.

\section{Quelle est votre spécialisation?}

En principe, je me vois comme un prestataire de base psychothérapeutique général. Je n'ai pas de spécialisations spécifiques à un trouble mental. J'aime particulièrement moccuper de mon travail en tant quanalyste dans mon groupe de psychothérapie ambulatoire ; un métier que je dois au séminaire pour l'analyse de groupe de Zurich.

Vous sentez-vous satisfait de votre situation professionnelle?

Oui, beaucoup. J'ai eu la chance de pouvoir exercer ma profession indépendante au bon moment. Je sais qu'aujourd'hui, les conditions d'une pratique professionnelle indépendante sont considérablement plus difficiles.

Y a-t-il quelque chose que vous souhaitez faire différemment?

Bien entendu, il serait souhaitable que le travail des psychothérapeutes psychologues soit beaucoup plus valorisé politiquement, socialement et économiquement. Mais ceci n'est probablement que pour le prix d'une compréhension médico-centrée croissante de la psychothérapie. Cependant, je considère que ce prix est trop élevé. En outre, le processus d'accréditation des cours de formation de psychothérapie est parfois difficile à comprendre, contradictoire et apparemment tacite selon les intérêts académiques.

La réglementation de la psychothérapie dans les professions relevant du droit fédéral de la psychologie contredit également les nombreuses expériences prouvées de processus de psychothérapie réussis. Car les facteurs principaux de ces psychothérapies peuvent être trouvées dans de bonnes relations, et cela nécessite un développement personnel sérieux des thérapeutes et et l'absence de prérequis de diplômes universitaires exclusivement en psychologie. Malheureusement, l'ancienne formation pluridisciplinaire d'accès à la psychothérapie a été fermée.

\section{$Y$ at-il quelque chose que vous souhaitez de votre association ASP?}

Bien entendu, l'ASP doit œuvrer dans l'intérêt de ses membres. Et ces intérêts se situent dans 
l'amélioration des conditions-cadres politiques, sociales et économiques pour la pratique professionnelle des psychothérapeutes en exercice. Cependant, penser à une compréhension de la psychothérapie de haute qualité ne doit pas être négligé et sacrifié aux intérêts professionnels de la pratique des psychothérapeutes. L'intérêt curatif de la psychothérapie, c'est-à-dire la compréhension de la psychothérapie comme remède, est sans aucun doute la publicité efficace à représenter. Cependant, l'intérêt émancipateur de la psychothérapie, cest-à-dire la compréhension de la psychothérapie comme un développement de la personnalité avec ses effets sur les relations de couple, les familles, les groupes et les organisations ne doit pas être oublié. Le congrès bien documenté en novembre 2014, intitulé « Émancipons-nous nous-mêmes! ", a tenté de prendre de nouvelles mesures dans cette direction.

Vous sentez-vous représenté et apprécié dans votre association professionnelle ASP ?

Oui.

Quel serait votre objectif si vous faisiez partie du Conseil d'administration de l'ASP ?

Comme indiqué ci-dessus, je me soucierais de la remise à jour, de lapprofondissement et de la diffusion de l'intérêt émancipateur de la psychothérapie, ainsi que du développement ultérieur $\mathrm{du}$ concept de formation continue "ASP Integral». Je considère toujours l'idée d'un programme de formation continue interscolaire propre à l'ASP comportant des structures méthodologiques intégrales à être prospectifs, puisque la préservation de la diversité des méthodes est toujours un objectif central de la philosophie de la Charte pour la psychothérapie de l'ASP. Avec de bonnes raisons. Parce qu'il est bien connu qu'un triple ajustement est nécessaire pour lancer des psychothérapies réussies : l'ajustement personnel entre le patient et le thérapeute, l'ajustement entre le thérapeute et la méthode et l'ajustement entre le patient et la méthode. Pour qu'il soit possible de trouver de tels ajustements, il faut une grande variété de méthodes et pas seulement deux ou trois courants principaux.

De plus, l'adhésion à une fédération de professionnels perd de son importance en raison d'une reconnaissance fédérale des psychothérapeutes. C'est pourquoi le concept d'un pool de formation continue au sein de lassociation professionnelle de l'ASP avec des parties interscolaires, mais aussi l'approfondissement intégral spécifique à la méthode pour les étudiants, constituent la meilleure publicité afin de rester fidèle à l'ASP comme une association professionnelle après avoir obtenu son diplôme.

Vous avez été impliqué dans l'ASP ou la Charte Suisse de la psychothérapie depuis de nombreuses années, y compris en tant que président de la Commission pour l'assurance qualité. Rétrospectivement, quels sont les points saillants de cette activité ?

La base de certains faits saillants a toujours été la coopération agréable avec les collègues, tant au sein de la Commission de lassurance qualité que du Conseil d’administration de la Charte. J'ai également trouvé enrichissantes, les rencontres avec les différents délégués des institutions de la charte dans les colloques et les discussions lors des revues dans les institutions et les associations. En règle générale, il était possible de donner des conseils utiles dans des " homelands » très différents. Chaque fois que la correspondance entre linstitution de formation continue et lobjectif de la charte était juste, une bonne relation entre les représentants des institutions et les membres de la Commission de révision pourrait devenir fructueuse.

Lapprobation unanime du concept de qualité développé depuis longtemps est devenu un point culminant, en partie parce que nous avons anticipé le règlement d'accréditation du gouvernement fédéral d'une manière prospective. En outre, lorganisation du Congrès "Émancipons-nous nous-mêmes ! ", qui portait sur l'impact émancipateur des autres disciplines de l'enseignement supérieur, a également été un événement réussi. Et troisièmement, je considère aussi que le travail au Conseil de formation de létude du PAPS est un succès, bien que dans notre domaine des conseillers différemment ancrés, ne parvenaient que faiblement à un bon accord des valeurs du diagnostic psychodynamique opérationalisé appliqué (Operationalisierte Psychodynamische Diagnostik - OPD) (cf. Müller-Locher, 2016).

Quelle serait votre situation souhaitée dans le contexte politique donné pour les psychothérapeutes?

Il est difficile de répondre, parce que mes souhaits visent avant tout à changer le climat politique. 
Bien entendu, les initiatives communes de tous les psychothérapeutes reconnus seraient plus efficaces que les conflits de blocage du changement entre psychologues et psychothérapeutes. Un bon accord entre les psychothérapeutes psychologiques et médicaux en fait également partie. Après tout, il s'agit d'améliorer les soins psychosociaux pour les personnes souffrant émotionnellement, ce qui ne devrait tolérer aucune lutte de tranchée obstructive ni de dispute de soi-disant prestataires de services. Et en outre, une telle connexion nécessite une réflexion sur la question de la réussite de la vie humaine et donc sur la relation entre émancipation éclairée et " santé » et/ou émancipation en tant que liberté et santé en tant que finitude durable. En d'autres termes, la psychothérapie a besoin de plus de philosophie et de politique et moins de théologie et désotérisme.

Quelle est votre vision dans votre vie professionnelle?

Puisque je suis personnellement très satisfait de ma situation professionnelle, je peux seulement dire que je continue comme avant. Quelques années de travail agréable en tant que psychothérapeute psychodynamique et superviseur, à temps partiel, bien entendu.

\section{Littérature}

Müller-Locher, P. (2011). Das Dilemma der Psychotherapie-Weiterbildung in der Schweiz - Erfahrungen und Reflexionen eines Insiders. In B. Bucher, T. Hagmann, R. Kuhn \& G. Thomann (Hrsg.), Resonanz - Gestalten von Organisationen in flüchtigen Zeiten, Bd. 2: Loyalität (S. 121-138). Bern: hep.

Müller-Locher, P. (2016). Der OPD-Ratingprozess. Persönliche Erfahrungen und Einsichten. In A. von Wyl, V. Tschuschke, A. Crameri, M. Koemeda-Lutz \& P. Schulthess (Hrsg.), Was wirkt in der Psychotherapie? Ergebnisse der Praxisstudie ambulante Psychotherapie zu 10 unterschiedlichen Verfahren (S. 51-63). Gießen: Psychosozial-Verlag.

o.A. (2014). «Emanzipieren wir uns!» Interdisziplinärer Kongress der Schweizer Charta für Psychotherapie. 8. November, Volkshaus Zürich [YouTube].

\section{CONCERNANT LA PERSONNE}

Peter Müller-Locher, Dr. Phil., MSc, est un psychothérapeute de l'ASP reconnu par le gouvernement fédéral et qui travaille dans son propre cabinet psychothérapeutique à Zurich et à Horgen. Il a été membre de l'ASP depuis 1986 et a occupé divers rôles dans la Charte suisse de psychothérapie de 1993 à 2018.

L'entrevue a été réalisée par écrit par Peter Schulthess.

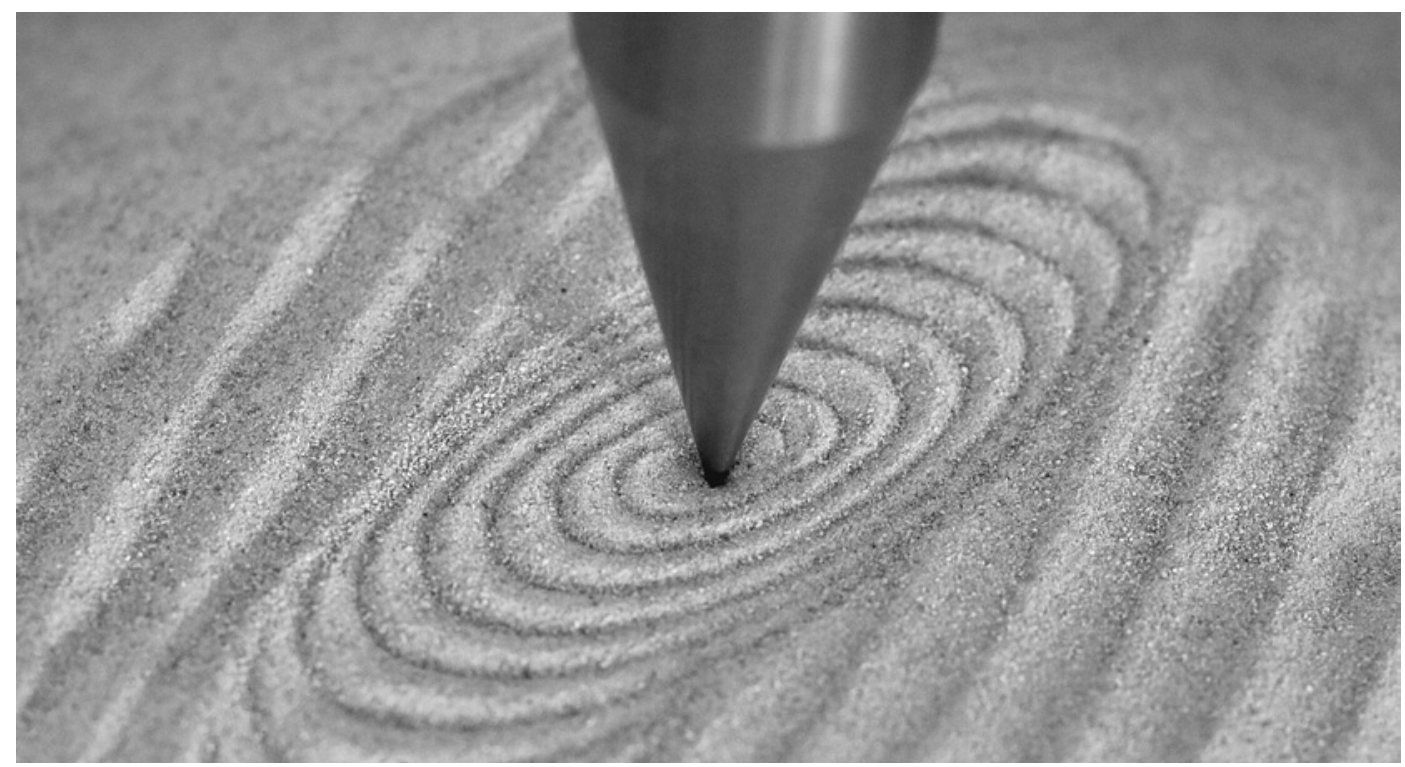

